\title{
Process and effects of a community intervention on malaria in rural Burkina Faso: randomized controlled trial
}

\author{
Bocar Kouyaté1, Florent Somé1, Albrecht Jahn², Boubacar Coulibaly1, \\ Jaran Eriksen³, Rainer Sauerborn², Lars Gustafsson³, Göran Tomson³, \\ Heiko Becher ${ }^{2}$ and Olaf Mueller*2
}

\begin{abstract}
Address: ${ }^{1}$ Centre de Recherche en Santé de Nouna, Nouna, Burkina Faso, Africa, ${ }^{2}$ Department of Tropical Hygiene and Public Health, University of Heidelberg, Germany and ${ }^{3}$ Karolinska Institute, Stockholm, Sweden

Email: Bocar Kouyaté - bkouyate@hotmail.com; Florent Somé - sieali@yahoo.fr; Albrecht Jahn - albrecht.jahn@cec.eu.int; Boubacar Coulibaly - boubacoulibaly@hotmail.com; Jaran Eriksen - jaran.eriksen@ki.se; Rainer Sauerborn - rainer.sauerborn@urz.uniheidelberg.de; Lars Gustafsson - lars-l.gustafsson@ki.se; Göran Tomson - goran.tomson@ki.se; Heiko Becher - heiko.becher@urz.uniheidelberg.de; Olaf Mueller* - olaf.mueller@urz.uni-heidelberg.de

* Corresponding author
\end{abstract}

Published: 25 March 2008

Malaria Journal 2008, 7:50 doi:10.1186/1475-2875-7-50

This article is available from: http://www.malariajournal.com/content/7///50

(c) 2008 Kouyaté et al; licensee BioMed Central Ltd.

This is an Open Access article distributed under the terms of the Creative Commons Attribution License (http://creativecommons.org/licenses/by/2.0), which permits unrestricted use, distribution, and reproduction in any medium, provided the original work is properly cited.

\begin{abstract}
Background: In the rural areas of sub-Saharan Africa, the majority of young children affected by malaria have no access to formal health services. Home treatment through mothers of febrile children supported by mother groups and local health workers has the potential to reduce malaria morbidity and mortality.

Methods: A cluster-randomized controlled effectiveness trial was implemented from 2002-2004 in a malaria endemic area of rural Burkina Faso. Six and seven villages were randomly assigned to the intervention and control arms respectively. Febrile children from intervention villages were treated with chloroquine (CQ) by their mothers, supported by local women group leaders. CQ was regularly supplied through a revolving fund from local health centres. The trial was evaluated through two cross-sectional surveys at baseline and after two years of intervention. The primary endpoint of the study was the proportion of moderate to severe anaemia in children aged 6-59 months. For assessment of the development of drug efficacy over time, an in vivo CQ efficacy study was nested into the trial. The study is registered under http://www.controlled-trials.com (ISRCTN 34I04704).
\end{abstract}

Results: The intervention was shown to be feasible under program conditions and a total of 1.076 children and 999 children were evaluated at baseline and follow-up time points respectively. Self-reported CQ treatment of fever episodes at home as well as referrals to health centres increased over the study period. At follow-up, CQ was detected in the blood of high proportions of intervention and control children. Compared to baseline findings, the prevalence of anaemia ( $29 \%$ vs $16 \%, p<0.000 I)$ and malaria parameters such as prevalence of $P$. falciparum parasitaemia, fever and palpable spleens was lower at follow-up but there were no differences between the intervention and control group. CQ efficacy decreased over the study period but this was not associated with the intervention.

Discussion: The decreasing prevalence of malaria morbidity including anaemia over the study period can be explained by an overall increase of malaria prevention and treatment activities in the study area. The lack of effectiveness of the intervention was likely caused by contamination, pre-existing differences in the coverage of malaria treatment in both study groups and an unexpectedly rapid increase of resistance against $C Q$, the first-line treatment drug at the time of the study. 


\section{Background}

At least one million annual malaria deaths occur among young children in rural sub-Saharan Africa (SSA). Most of these deaths are in populations with little access to health services [1-4] In such areas, home treatment with chloroquine (CQ), antipyretics and traditional remedies is the most frequent response of caretakers to fever episodes in children [4-6]. However, due to the increasing resistance against CQ in most countries in SSA together with limited access to modern health services, poor quality of such services, low compliance with treatment schemes and poor quality of drugs sold at markets, the community effectiveness of malaria treatment is very low [6-13].

As malaria treatment provided through formal health services is currently not a sufficiently effective strategy for malaria control in rural SSA, interventions aiming at improving malaria home treatment by main caretakers, usually the mothers, may be considered as a complementary strategy. There is some evidence that improved home management of malaria in young children of SSA will result in earlier and more effective treatment with consequently reduced morbidity and mortality $[14,15]$.

This project is an EU INCO-DEV funded collaboration between the Heidelberg University (Germany), Karolinska Institute (Sweden), Muhimbili University College of Health Sciences (Tanzania) and Centre de Recherche en Santé de Nouna (Burkina Faso) called MAMOP project (Improving the management of childhood MAlaria: an experiment to bridge the gap between MOthers and health care Providers). It is a controlled malaria community intervention with a pre-post design conducted in rural Burkina Faso and Tanzania in 2002 - 2004. The overall objective of the MAMOP study was to evaluate the feasibility and effectiveness of an intervention aimed at improving case management of malaria in underfive children through primary caretakers in collaboration with local women groups and existing health centres.

\section{Methods \\ Study area}

The study was implemented in the rural part of the research zone of the Centre de Recherche en Santé de Nouna (CRSN) in Nouna Health District, north-western Burkina Faso (Figure 1). The Nouna area is a dry orchard savannah, populated mainly by subsistence farmers of different ethnic groups. Malaria is holoendemic but highly seasonal, and the transmission intensity varies between 100 and 1000 infective bites per person and year between study villages $[16,17]$. Formal health services in the study area are provided by a limited number of rural health centres and the district hospital in Nouna town [18]. Villagebased health centres are usually equipped with two nurses and one mid-wife and do outreach work in the surround- ing 7-10 villages under their responsibility. Malaria control is mainly based on home treatment with CQ, which has been shown to be still sufficiently effective in 2001, and on malaria prophylaxis for pregnant women $[4,19,20]$. Untreated mosquito nets have been used in the area for a long time, but insecticide-treated nets (ITN) were only recently introduced in the frame of an effectiveness study $[21,22]$. Communities in the study area have been shown to be quite well organized with regard to risk sharing mechanisms. In particular women groups with a focus on mutual agricultural support traditionally exist in all villages [23].

\section{Study design}

The study was designed as a cluster-randomized controlled effectiveness trial. Using the data base of the Nouna Demographic Surveillance System (DSS) [24], villages were selected by lottery (OM, AJ) until an approximate sample size of 1.200 households per study arm was achieved. As a result, six (Pa, Toni, Kemena, DenissaMossi, Boune, Lekuy) and seven (Bankoumani, Kamadena, Labarani, Barakuy, Dankoumana, Soulemana, Tissi) villages were randomly assigned to the intervention and control arms respectively (Figure 2).

The primary outcome of the study was the proportion of moderate to severe anaemia (haematocrit $\leq 24 \%$ ) in children aged 6 to 59 months. Secondary outcomes were prevalences of fever, malaria, of palpable spleens (Hackett score $\geq 2$ ), of other illnesses, mean species-specific number of blood films positive for malaria parasites, mean species-specific malaria parasite densities, mean haematocrit values and mean weight. Finally, in vivo CQ efficacy was measured in all study children with uncomplicated falciparum malaria at baseline and at follow-up using a modified version of the standard WHO protocol [25].

To be able to detect a $10 \%$ difference in anaemia prevalence between the intervention and control group with $80 \%$ power and at a significance level of $5 \%$, and assuming a prevalence in the control group of $20 \%$, and a conservative design factor of 2.5, 992 under-five children were required [26]. As the households were used as units of analysis and as around $80 \%$ of all households include an under-five child, about 1200 households had to be sampled.

\section{The intervention}

The intervention was targeted at three groups: health workers (nurses) from five peripheral health centres (Toni, Dara, Bourasso, Lekuy, Koro), women group leaders, and the main care takers (usually the mothers) of preschool children. The community members of the six intervention villages selected a total of 70 women group 


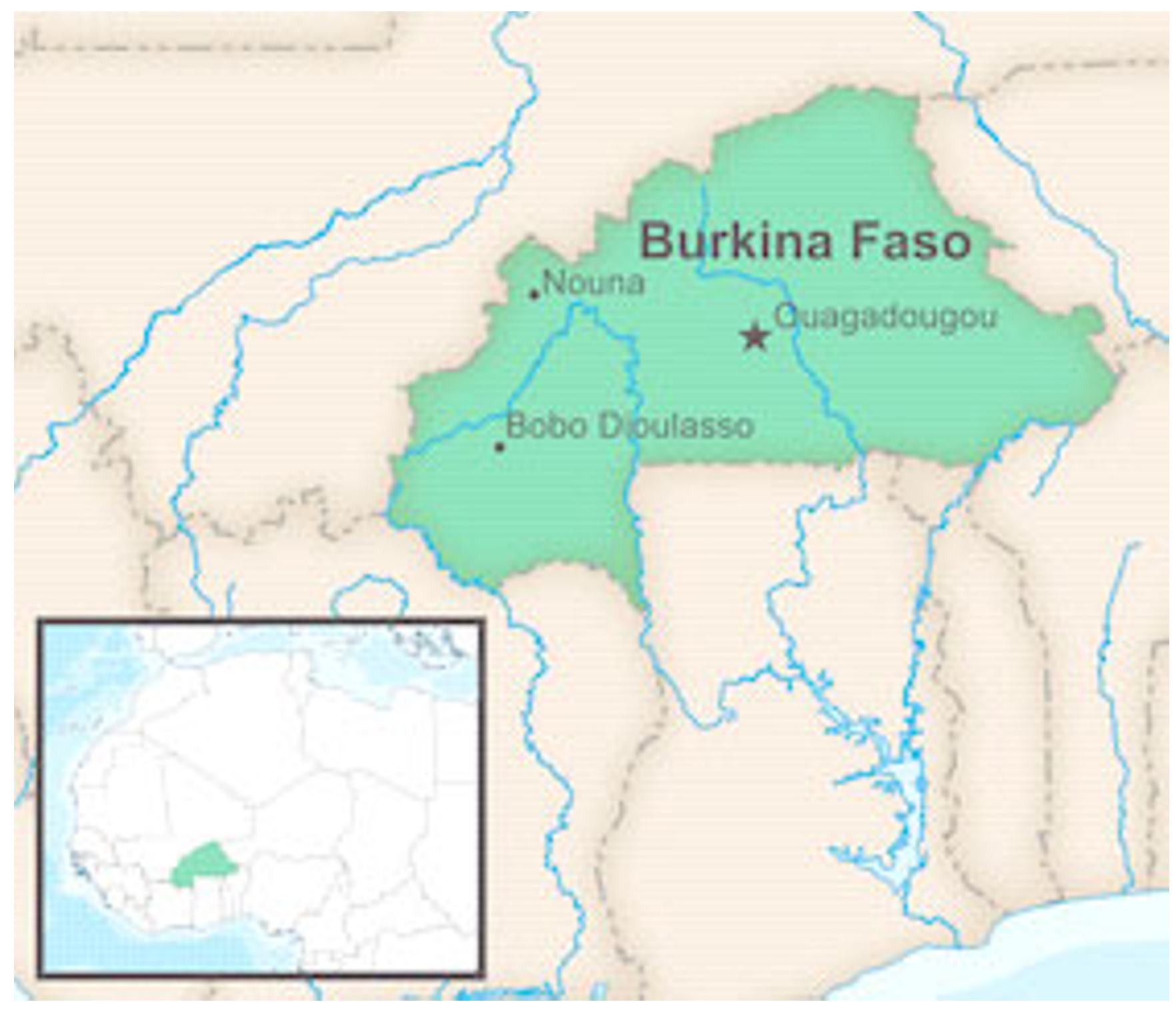

Figure I

Map of Burkina Faso.

leaders, two by sub-village (sub-village numbers ranged from 3-9 per village). Inclusion criteria for group leaders used by the communities were permanent residency in the sub-village, age 30-50 years, honesty, and respect by the community. The main components of the intervention were:

- Training of health staff, women group leaders and mothers

- Sensitisation of the communities

- Drug supply to women group leaders, revolving fund
- Supervision of health workers and women group leaders

- Intervention information system.

A five days training course for the health workers of participating health centres was conducted by one of the investigators (FS) together with the District Medical Officer and included an update on malaria case management and an introduction to adult non formal education methods. Afterwards, all women group leaders were trained in respective peripheral health centres by the health workers under the supervision of the investigators for two days on all relevant aspects of malaria knowledge and management including referral criteria. The training 


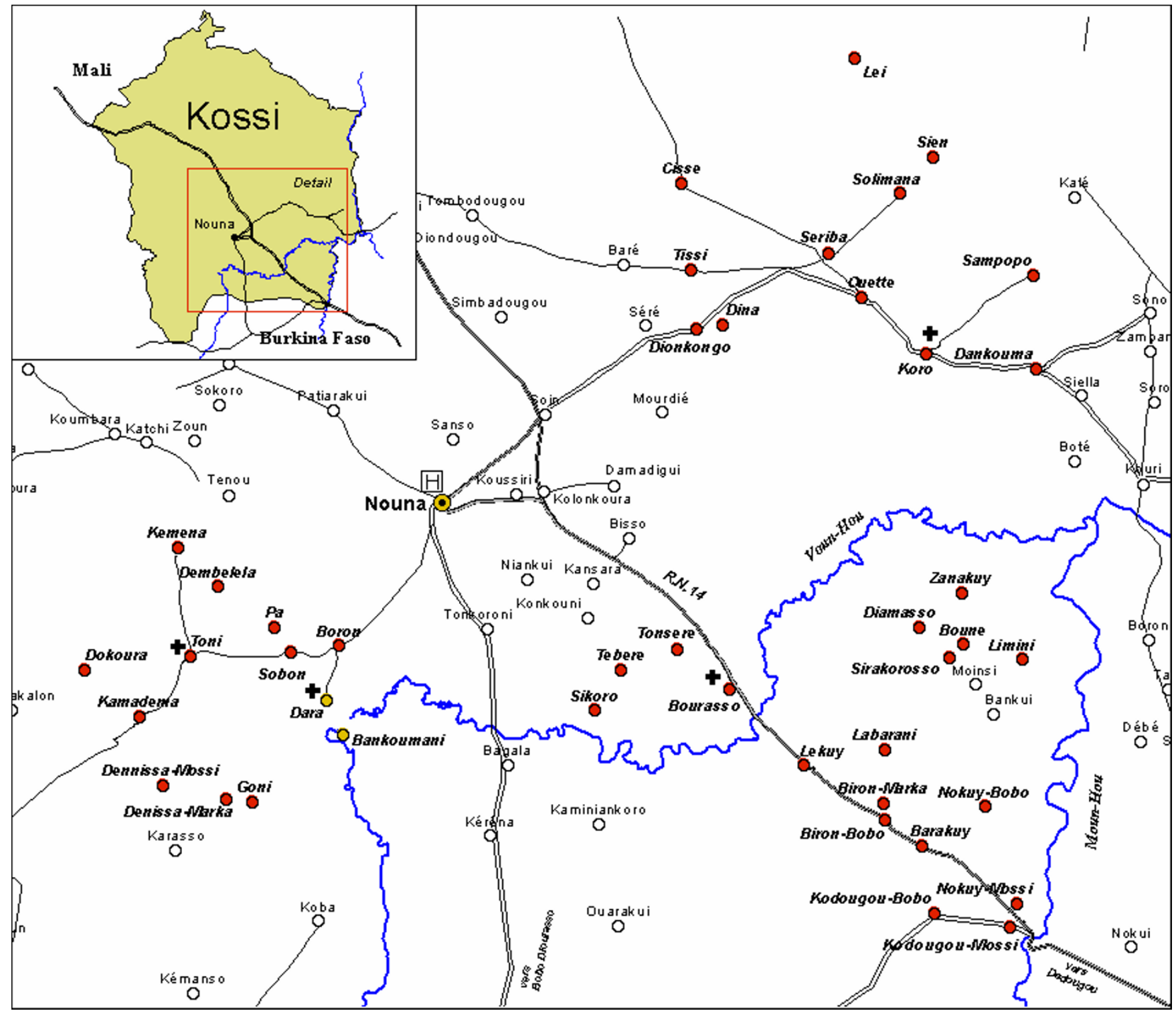

Figure 2

Map of the Nouna study area.

included discussions as well as practical sessions using locally produced pictorial sensitisation material and role play, with one refresher course over the study period (Figures $3,4,5)$. After this was completed, a sensitisation campaign was done in all intervention villages. Thereafter, the women group leaders under the supervision of their local health workers trained an average of 15 mothers for half a day in their sub-villages on correct malaria management.

All women group leaders were regularly provided with CQ and paracetamol from the essential drug stock of Nouna Health District. Pre-packed tablets have been shown to be better for ensuring compliance compared to loose tablets in African countries including Burkina Faso $[14,27,28]$. Thus, CQ and paracetamol were pre-packed in plastic bags in four age-specific doses $(0-6$ months, $7-11$ months, $1-3$ years, $4-5$ years) each with a specific colour and containing pictorial guidelines according to national malaria treatment guidelines (Figures 3, 4, Table 1).

Mothers with febrile children were advised to go to the house of women group leaders for early treatment. Children were treated with a total dose of $25 \mathrm{mg} / \mathrm{kg}$ CQ over a period of three days (first and second day: $10 \mathrm{mg} / \mathrm{kg}$, third day: $5 \mathrm{mg} / \mathrm{kg}$ ). In addition, all children received standard doses of paracetamol (10 mg/kg every 12 hours) over the 


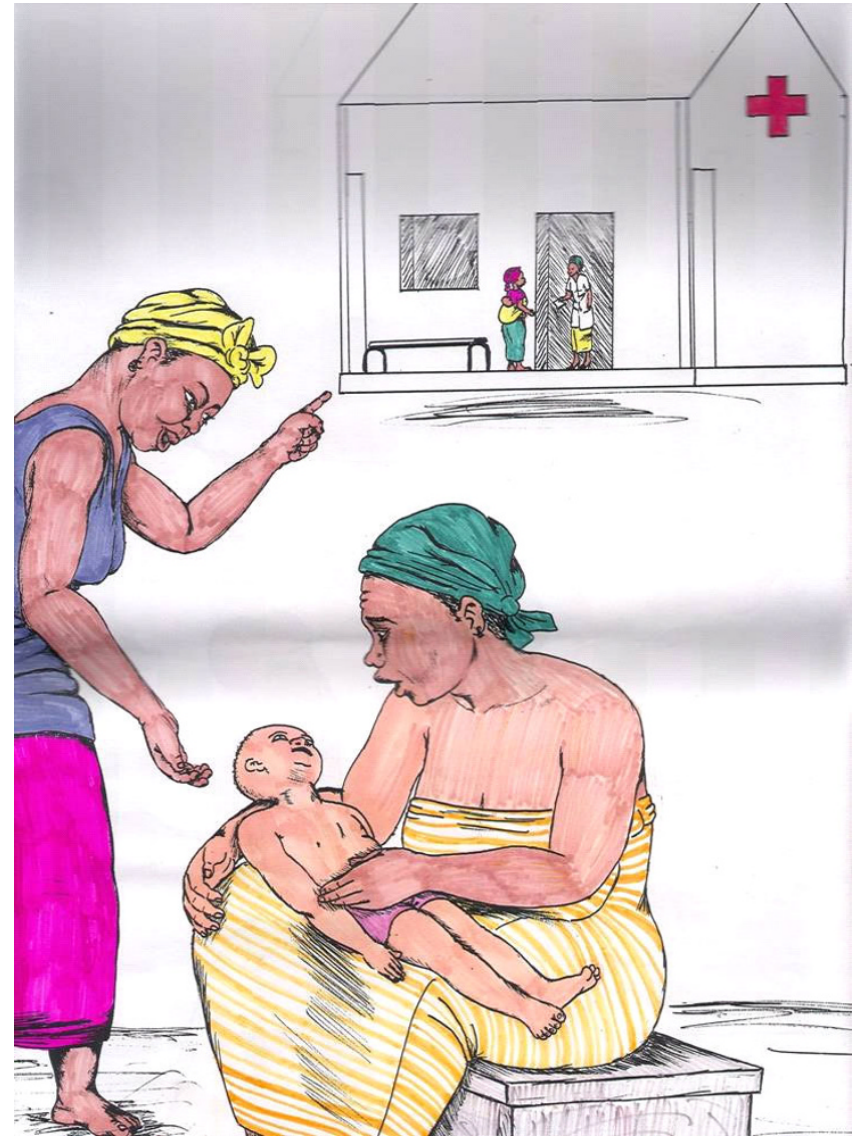

Figure 3

Pictoral chart example: referral of severely sick children to the health centre.

first two days. Women group leaders were advised to directly supervise the first dose of CQ and paracetamol, and to visit the sick child again on the second and third day. In case of danger signs at any time point or ongoing fever at the end of the treatment time, the women group leaders had to refer the child to the health centre.

At the beginning of the intervention, a six months stock of drugs was provided free of charge to the women group leaders, who had to afterwards renew their stock by buying new drugs from the health workers. Drugs were sold by the women group leaders to the mothers/caretakers at prices which allowed them to make a small profit as an incentive (table 1). The health workers visited the sub villages monthly for supervision with a standardized checklist, collection of forms and for drug provision. Overall supervision and if necessary - specific support - was carried out by the investigators every month for the first three months and thereafter every three months.
The intervention was monitored through a specific intervention information system which used forms based on pictorial charts, which were filled in every month by the women group leaders (figure 5). The first form collected information on (1) the number of malaria cases managed, (2) the number of home visits done, (3) the number of cases referred to the health facilities, (4) the number of children who had visited the health facilities, and (5) the number of children who had died. The second form reported the time between illness onset and initiation of the treatment. The third form reported the number of prepackaged drug bags used for each age group every month.

Information about the process of the intervention in health facilities was gathered through specific data collection tools (supervision report, pre-packaged drugs inventory list) as well as through the routine health information system (health centres registers, patients clinical files, referrals and counter referrals sheets).

The intervention had been pre-tested in three villages outside the study area. For the main study, training of health staff and women group leaders took place from February until June 2003. The intervention itself was fully implemented from July 2003 until October 2004, thus covering two rainy seasons.

\section{Study evaluation}

A baseline survey took place in September/October 2002, and a follow-up survey in September/October 2004.

All households with children below 5 years of age were included into the two surveys. In households with more than one eligible child, the survey child was chosen by lottery. All survey children were examined by the study physician (FS). Children found ill during the surveys were treated according to national guidelines. Those fulfilling the criteria for uncomplicated falciparum malaria (fever + $\geq 5.000$ Plasmodium falciparum parasites per $\mu \mathrm{l}$ ) were followed up for in vivo drug efficacy testing over a two weeks period. The WHO definitions for early treatment failure (ETF), late clinical failure (LCF), late parasitological failure (LPF) and adequate clinical and parasitological response (ACPR) were applied [25].

All children included into the in vivo drug efficacy study received standard doses of CQ and paracetamol over three days. Administration of study medications was directly supervised by the study team on day 0 and by villagebased field workers on day 1 and day 2 . In case of vomiting within one hour after the study medication, the medication was repeated. No drugs were allowed as concomitant treatment. Over the 14 days follow-up period, study children were seen daily by the field workers, who checked them for danger signs, measured their 

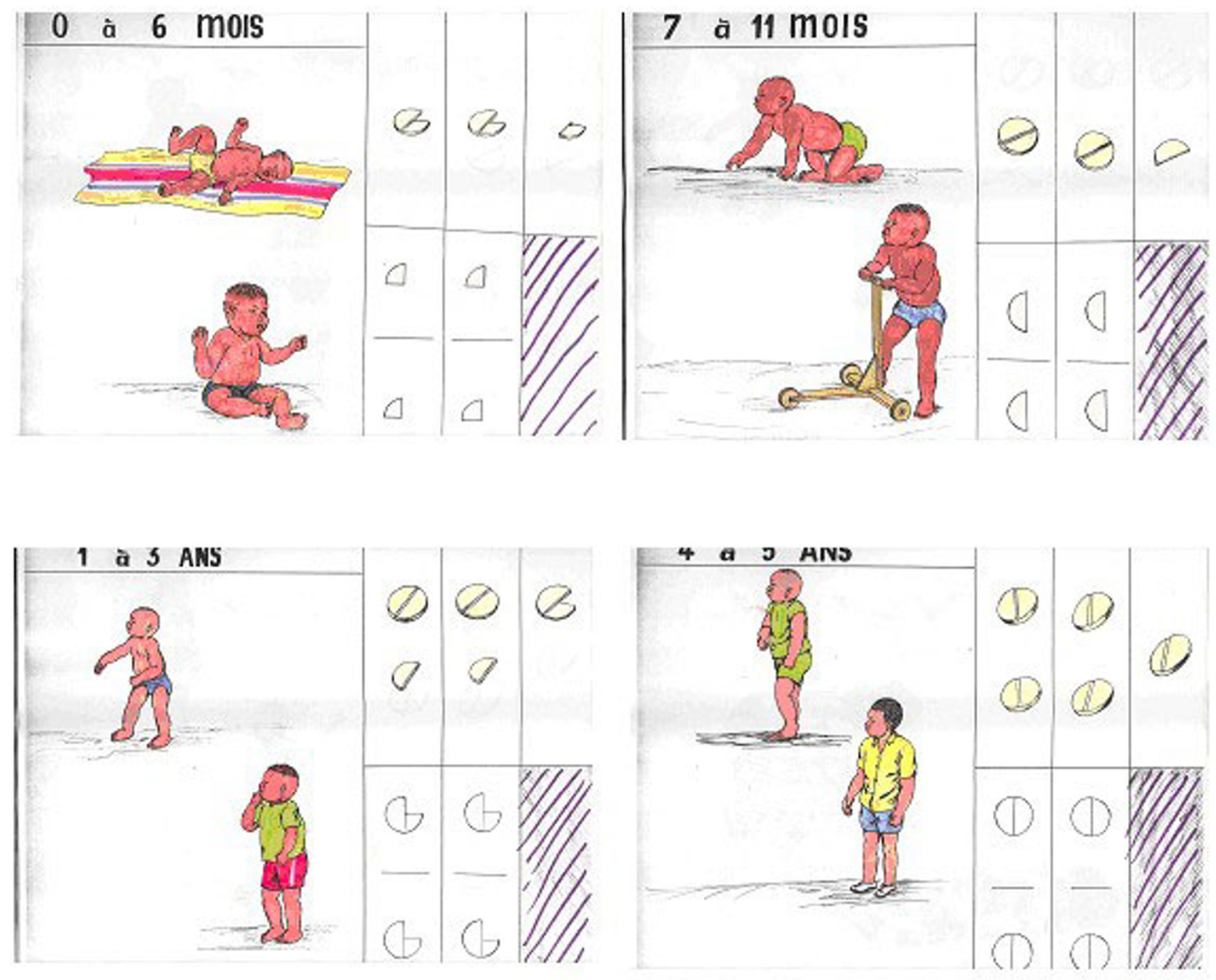

\section{Figure 4}

Pictoral chloroquine treatment guidelines by age group.

axillary temperature with a thermometer, and took finger prick blood samples. In case of clinical or parasitological failure, children were given single-dose pyrimethaminesulfadoxine rescue treatment (taken from the essential drug store of Nouna Health District) according to national guidelines. Children with danger signs were referred to the health centre.

Treatment procedures were followed through documentation of self-reported behaviour during surveys as well as through CQ determination in blood samples.

\section{Laboratory investigations}

From all children seen at the baseline and follow-up survey, a finger-prick blood sample was taken. From this, thin and thick blood smears were prepared for malaria diagnosis while anaemia determination was done through measurement of haematocrit values with a portable microhaematocrit centrifuge (Compur Microspin, Bayer Diagnostics, Germany).

For those children included into the CQ efficacy study, additional finger-prick blood samples were taken on day 2 , day 3 , day 7 , day 14 , and on any other day the child presented with symptoms during follow up according to standard procedures [25]. Moreover, pre-treatment filter 


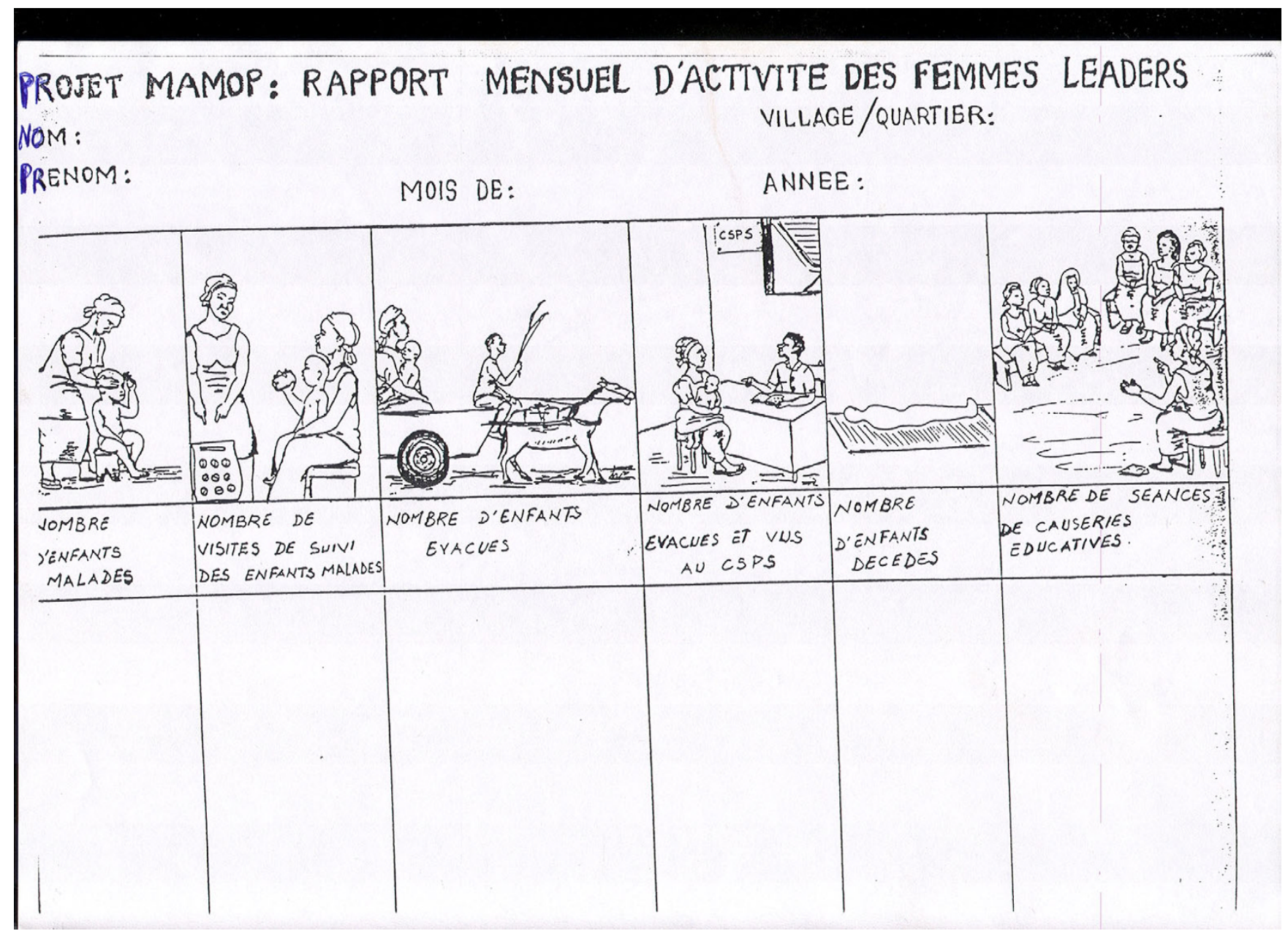

Figure 5

Pictoral chart example: intervention information system.

paper blood samples were taken from every child and randomly distributed in equal proportions for determination of chloroquine content and chloroquine resistance markers. Concentration of chloroquine and its metabolite desethylchloroquine were measured using high pressure liquid chromatography at Karolinska Institute in Stockholm.

Blood films were kept in closed slide boxes until they were transported to the CRSN laboratory in Nouna town. After

Table I: Drug package (chloroquine + paracetamol) prices by provider and age group

\begin{tabular}{lll}
\hline Age groups & Prices to women leaders & Prices to caretakers \\
\hline $0-6$ months & I5 FCFA (0.03 US \$) & 30 FCFA (0.06 US \$) \\
$7-11$ months & 20 FCFA (0.04 US \$) & 40 FCFA $(0.08$ US \$) \\
$1-3$ years & 30 FCFA (0.06 US \$) & 60 FCFA $(0.12$ US \$) \\
$4-5$ years & 40 FCFA (0.08 US \$) & 80 FCFA $(0.16$ US \$) \\
\hline
\end{tabular}

Giemsa-staining, all films were examined by experienced laboratory technicians. Thick and thin blood films were analysed for the species-specific parasite density per $\mu$ l by counting against 200 white blood cells and multiplying by 50 . Slides were declared negative if no parasites were seen in 400 fields on the thick film. For quality control a $10 \%$ random sample of blood films is regularly cross checked at the laboratory of the Heidelberg School of Tropical Medicine [21].

\section{Statistical analysis}

To assess the effect of the intervention, logistic regression was used. In separate models, anaemia (haematocrit $\leq$ $24 \%$ ), fever $\left(\geq 37.5^{\circ} \mathrm{C}\right)$, spleen enlargement (Hackett score $\geq 2$ ), clinical malaria (fever $+\geq 5000$ parasites $/ \mu \mathrm{l}$ ), and malaria parasitaemia ( $>0$ parasites $/ \mu \mathrm{l}$ ) were considered as binary outcomes. Age (continuous), weight (continuous) and ethnic group (4 groups: Bobo, Dafing, Mossi, other) were considered as co-variables for adjust- 


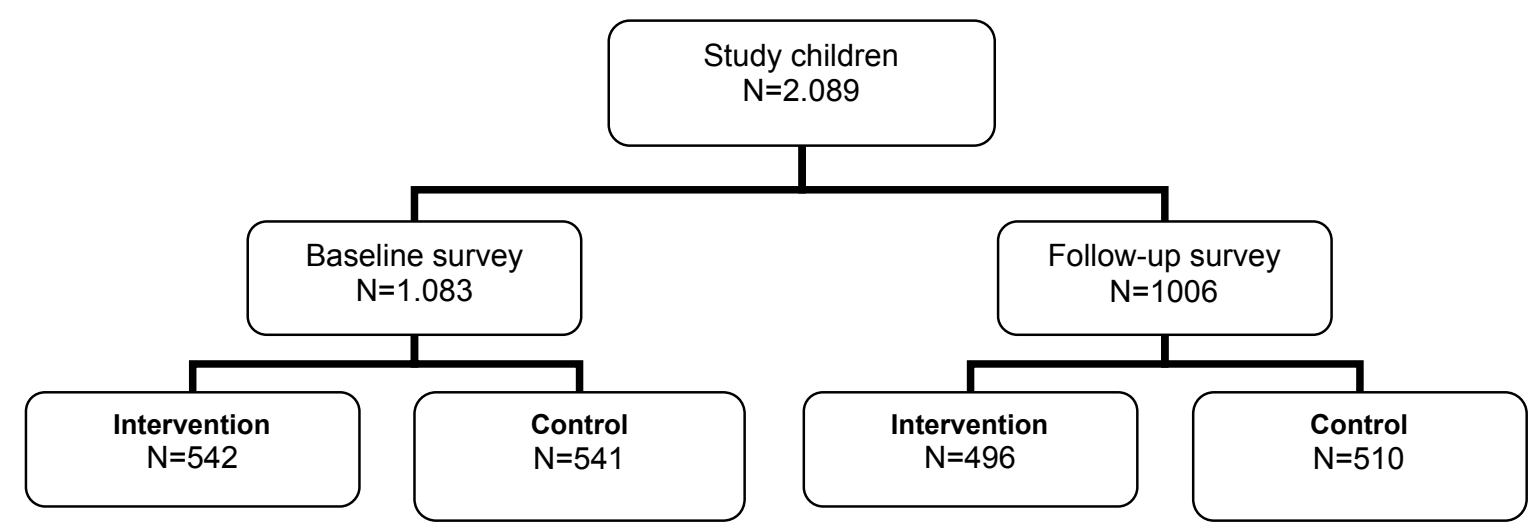

Figure 6

MAMOP study flow chart.

ment in the model. The distribution of the outcome variables in intervention and control group was compared at baseline to check adequacy of randomization. Fisher exact test was used to compare rates. The analysis was performed with SAS release 8.02 (SAS ${ }^{\circledast}$ Institute Inc, Cary, NC, USA).

\section{Ethical aspects}

The protocol was approved by the local Ethics Committee in Burkina Faso. Community consent was sought during village meetings in all study villages. During the following visits to individual households, caretakers were asked for their oral consent after having received detailed information from the study physician about all risks and benefits of the study. They were clearly informed that they could withdraw from the study at any time and without disadvantage. All children in the specified age group found to be ill during cross-sectional surveys or during follow-up in case of the CQ resistance study received free treatment in the village or were referred to Nouna hospital if indicated.

\section{Results \\ Study group characteristics}

A total of 1,083 children (542 from intervention and 541 from control villages) and a total of 1006 children (496 from intervention and 510 from control villages) were included into the study at baseline and follow-up time points respectively (Figure 6).

Table 2 shows demographic characteristics of children from both study groups at baseline and follow-up time points. Comparing children from intervention and control clusters at baseline and follow-up, there were no significant differences with regard to age and sex. However, intervention and control clusters differed much with regard to the presence of the Bobo and Marka ethnicity. Comparing children from the intervention and control clusters over time, the samples did not differ significantly with the exception of follow-up children having been slightly older in both groups at follow-up.

Table 2: Demographic, clinical and parasitological characteristics of study children at baseline and follow-up time points.

\begin{tabular}{lcccc}
\hline & Baseline survey & Baseline survey & Follow-up survey & Follow-up survey \\
\hline & Intervention $(n=542)$ & Control $(n=54 I)$ & Intervention $(n=496)$ & Control $(n=5 I 0)$ \\
\hline Ethnicity (\%) & & & & \\
Marka & $99(18)$ & $312(58)$ & $100(20)$ & $305(60)$ \\
Bobo & $301(56)$ & $116(21)$ & $280(56)$ & $96(19)$ \\
Peulh & $33(6)$ & $35(6)$ & $17(3)$ & $35(7)$ \\
Mossi & $81(15)$ & $51(9)$ & $13(16)$ & $44(9)$ \\
Samo & $18(3)$ & $21(4)$ & $7(1)$ & $26(5)$ \\
Other & $10(2)$ & $6(1)$ & 35 & $4(1)$ \\
Median age (months) & 31 & 30 & $(5-60)$ & 35 \\
Median age (range) & $(5-56)$ & $(4-56)$ & $238 / 258$ & $(5-60)$ \\
Male/female & $285 / 257$ & $266 / 275$ & & $267 / 243$
\end{tabular}




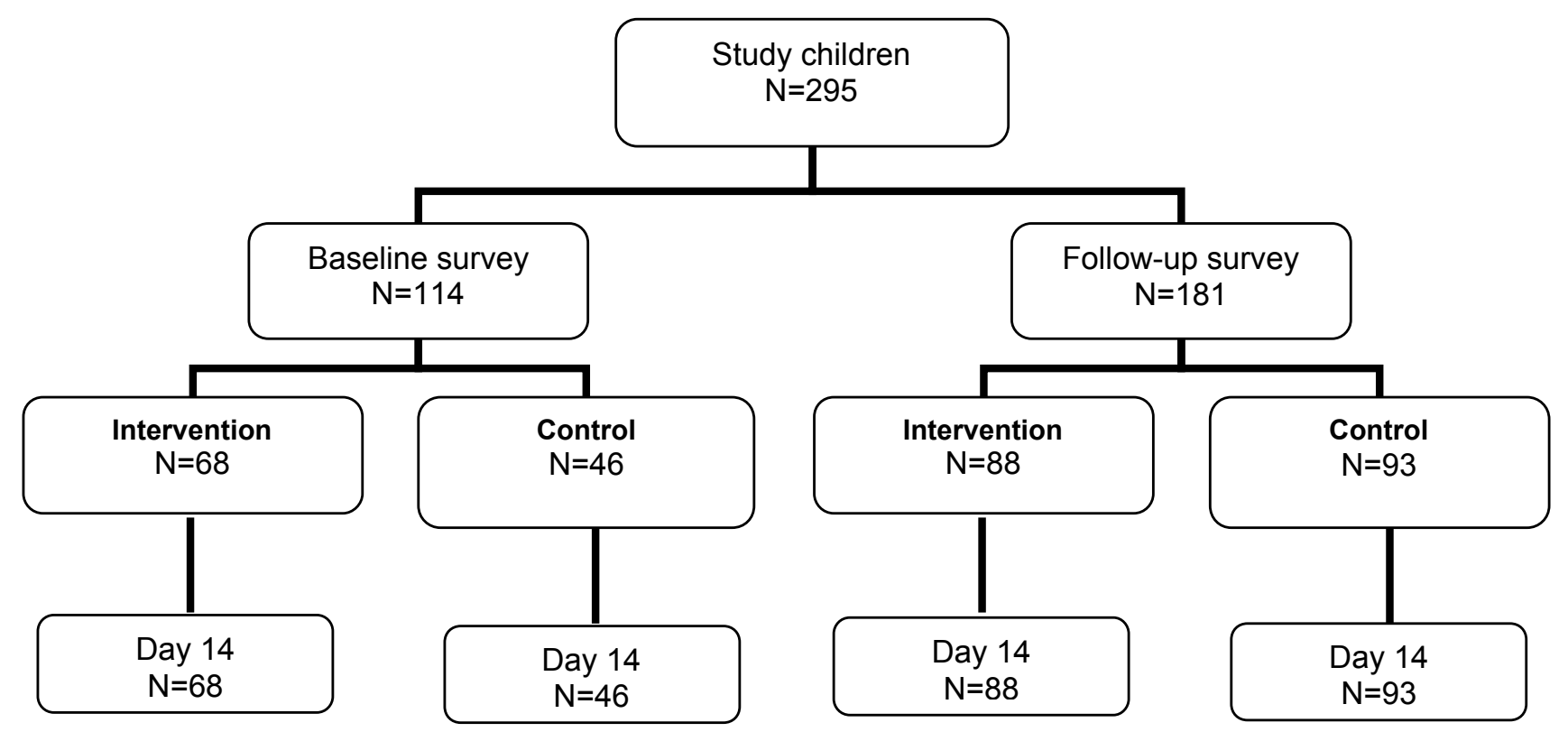

Figure 7

Chloroquine efficacy study flow chart.

A total of 114 children ( 68 from intervention and 46 from control villages) and a total of 181 children (88 from intervention and 93 from control villages) were included into the CQ efficacy study at baseline and follow-up time points respectively (Figure 7). The difference in number of children from this sub-study at baseline and follow-up is explained by the fact that for logistical reasons, the recruitment of children at baseline was only in four villages (two intervention, two control), while at follow-up, it was in nine villages (five intervention, four control). All four villages sampled at baseline were again sampled at followup. There were no major differences in baseline demographic, clinical or parasitological characteristics between survey children (Table 3 ). All study children completed the 14 days follow-up.

\section{Compliance with the intervention}

Some 12.500 pre-packaged malaria treatments were sold in the intervention villages over the period July 2003 until October 2004. Table 4 shows the data on self-reported compliance in study children during baseline and followup surveys. The number of self-reported fever episodes (fever during last two days) was lower during the baseline survey compared to the follow-up survey (29\% vs 52\%), without differences between study groups. At baseline, more children in the control villages compared to intervention villages reported having treated these fever episodes with CQ at home (64\% vs $35 \%)$, but this was reversed at follow-up $(60 \%$ vs $72 \%)$. At follow-up, women group leaders were reportedly consulted for the management of CQ home treatment episodes in $88 \%$ in the intervention group but also in $9 \%$ of the control group. The proportion of fever episodes treated at formal health services was very low at baseline and increased over

Table 3: Demographic, clinical and parasitological characteristics of children included into the chloroquine efficacy study at baseline and follow-up time points

\begin{tabular}{lllll}
\hline & Baseline survey & Baseline survey & Follow-up survey & Follow-up survey \\
\hline & Intervention $(n=68)$ & Control $(n=46)$ & Intervention $(n=88)$ & Control $(n=93)$ \\
\hline Mean age, range (months) & $30.1 ; 6-58$ & $33.5 ; 12-60$ & $25.1 ; 6-58$ & $27.7 ; 6-58$ \\
Male & 35 & 21 & 46 & 45 \\
Mean weight, range $(\mathrm{kg})$ & $11.1 ; 6.5-16.9$ & $11.2 ; 5.9-17.3$ & $10.1 ; 5.9-18.3$ & $10.3 ; 5-16$ \\
Mean temperature, range $\left({ }^{\circ} \mathrm{C}\right)$ & $38.0 ; 37.5-39.5$ & $38.0 ; 37.5-39.4$ & $38.1 ; 37.5-40.1$ & $38.2 ; 37.5-40.5$ \\
Mean number P. falciparum trophozoites, range & $19.826,5.000-67.000$ & $24.541 ; 5.000-158.000$ & $33.448 ; 5.000-174.500$ & $21.063 ; 5.000-101.000$
\end{tabular}


Table 4: Self-reported treatment procedure information in study children during baseline and follow-up surveys

\begin{tabular}{|c|c|c|c|c|}
\hline & Baseline survey & Baseline survey & Follow-up survey & Follow-up survey \\
\hline & Intervention $(n=542)$ & Control $(n=541)$ & Intervention $(n=496)$ & Control $(n=510)$ \\
\hline Fever last 2 days (\%) & $179 / 542(33)$ & $|32 / 54|(24)$ & $24 I / 496(49)$ & $282 / 510(55)$ \\
\hline \multicolumn{5}{|l|}{ Treatment of fever episode } \\
\hline - with CQ at home (\%) & $62 / 179(35)$ & $85 / 132(64)$ & $|72 / 24|(72)$ & $168 / 282(60)$ \\
\hline - at health centre (\%) & $4 / 179(2)$ & $2 / 132(2)$ & $36 / 241(15)$ & $23 / 282(8)$ \\
\hline Involvement of women group leaders in $\mathrm{CQ}$ treatment & - & - & $151 / 172(88)$ & $15 / 168(9)$ \\
\hline
\end{tabular}

the study period ( $2 \%$ vs $11 \%)$. There were no differences in this treatment pattern at baseline $(2 \%$ vs $2 \%)$, but the increase was higher in the intervention compared to the control villages during follow-up ( $15 \%$ vs $8 \%)$.

Due to technical problems, no measurements of $\mathrm{CQ}$ blood levels were possible during the baseline survey. During the follow-up survey, $\mathrm{CQ}$ was detected in the blood of $33 / 43(77 \%)$ and $37 / 43(86 \%)$ while the median CQ blood level (range) was $212 \mathrm{nmol} / \mathrm{L}$ (23-26.878) and 407 (23-13.734) $\mathrm{nmol} / \mathrm{L}$ in intervention and control group children respectively.

\section{Effects of the intervention on primary and secondary outcomes}

Table 5 gives the primary and secondary study outcomes except the CQ efficacy results. The prevalence of anaemia, fever, splenomegaly and $P$. falciparum parasitaemia but not of falciparum malaria was significantly lower during the follow-up compared to the baseline survey, but there were no significant differences between intervention and control group. A multivariate analysis showed no effect of the intervention on any of the outcome variables considered. However, there was a highly significant improvement regarding the prevalence of anaemia, fever, malaria,
P. falciparum parasitaemia and splenomegly at the followup survey independent of the intervention $(p<0.001)$.

The results of the in vivo CQ efficacy study are given in table 6 . At baseline, the overall day 14 ACPR was 76/114 $(67 \%)$, without differences between children from intervention and control villages. At follow-up, an ACPR occurred in $46 / 88$ (52\%) of intervention group children and in $41 / 93(44 \%)$ of control group children. After controlling for baseline variables, there was a significant effect of time (OR 0.4, 95\% CI 0.2-0.7), but not of the intervention (OR 1.4, 95\% CI 0.8-2.5) on ACPR.

\section{Discussion}

This study was designed as a cluster-randomized controlled trial with a sufficient sample size to show intervention effects of public health importance on malaria parameters. The intervention was shown to be feasible under programme conditions and the uptake was documented by an increase of malaria treatment with CQ in the intervention households and by an increase of referrals to the local health centres. Unfortunately, at baseline the use of CQ was higher in the control compared to the intervention villages which points to the fact that intervention and control area differed with regard to treatment behaviour. However, although CQ treatment increased in the inter-

Table 5: Effects of the intervention on study outcomes

\begin{tabular}{|c|c|c|c|c|c|}
\hline & Baseline survey & Baseline survey & Follow-up survey & Follow-up survey & $p^{\S}$ \\
\hline & Intervention $(\mathrm{n}=542)$ & Control $(n=541)$ & Intervention $(n=496)$ & Control $(n=510)$ & \\
\hline Anaemia* (\%) & $152(28)$ & $162(30)$ & $83(17)$ & $74(15)$ & 0.32 \\
\hline Fever prevalence ${ }^{* *}(\%)$ & $201(37)$ & $189(35)$ & $143(29)$ & $142(28)$ & 0.40 \\
\hline Falciparum malaria prevalence*** (\%) & $87(16)$ & $85(16)$ & $64(13)$ & $71(14)$ & 0.45 \\
\hline Spleen enlargement ${ }^{* * * *}(\%)$ & $108(20)$ & $79(15)$ & $22(4)$ & $19(4)$ & 0.08 \\
\hline P. falciparum parasitaemia (\%) & $455(84)$ & $438(8 I)$ & $379(76)$ & $366(72)$ & 0.05 \\
\hline Range & $80-100.000$ & $40-129.000$ & $50-180.000$ & $100-108.000$ & \\
\hline Median P. falciparum & 5.000 & 5.000 & 3.000 & 3.000 & \\
\hline Median haematocrit (range) & $28(12-40)$ & $28(14-40)$ & $30(16-42)$ & $30(10-40)$ & \\
\hline Median weight $(\mathrm{kg})$ & 11.2 & 11.1 & II.I & 11.6 & \\
\hline
\end{tabular}

$*$ haematocrit $\leq 24 \%, * * \geq 37.5^{\circ} \mathrm{C}, * * * \geq 37.5^{\circ} \mathrm{C}+\geq 5.000$ parasites $/ \mu \mathrm{l}$, ****Hackett $\geq 2, \S \mathrm{p}$-value for effect of intervention on study outcomes adjusted for time, age, weight and ethnic group 
Table 6: Chloroquine efficacy in young children of rural Burkina Faso in intervention and control communities

\begin{tabular}{|c|c|c|c|c|c|}
\hline & Baseline survey & Baseline survey & Follow-up survey & Follow-up survey & p-value* \\
\hline & Intervention $(n=68)$ & Control $(n=46)$ & Intervention $(\mathrm{n}=88)$ & Control $(n=93)$ & \\
\hline ETF (\%) & $8(12)$ & $3(7)$ & $13(15)$ & $12(13)$ & 0.88 \\
\hline LCF (\%) & $4(6)$ & $5(\mathrm{II})$ & $4(5)$ & $13(14)$ & 0.04 \\
\hline LPF (\%) & $11(16)$ & $7(15)$ & $25(28)$ & $27(29)$ & 0.99 \\
\hline ACPR (\%) & $45(66)$ & $31(67)$ & $46(52)$ & $41(44)$ & 0.30 \\
\hline
\end{tabular}

ETF = Early Treatment Failure; LCF = Late Clinical Failure; LPF = Late Parasitological Failure; ACPR = Adequate Clinical and Parasitological response.

*Comparison of proportions at follow-up survey (Fisher exact test)

vention villages it was observed to a similar degree in the control villages. This is to a large degree explained by the high degree of pre-existing CQ treatment in control villages. Moreover, contamination could have occurred by communication between the villagers of both study arms but also by local health workers who partly were responsible for both intervention and control villages. Such an interpretation is also supported by the fact that some $10 \%$ of women in control villages also received support from women group leaders. A further explanation for the high degree of CQ treatment in the control villages is a possible contamination by a parallel community-based CQ-distribution project in the area. In this GTZ (Gesellschaft für Technische Zusammenarbeit) - supported reproductive health project, women of 18 villages neighbouring the MAMOP study area received contraceptives but also CQ through trained volunteers. This unforeseen additional contamination has likely contributed to making the control zone not a real control zone.

The main findings from the study were significant reductions in the prevalence of anaemia, P. falciparum parasitaemia and spleen enlargement over time, but without differences between study groups. The differences in the prevalence of anaemia and malaria parameters between the baseline survey in 2002 and the follow-up survey in 2004 are likely caused by a higher use of CQ in the study area. This hypothesis is supported by the finding of high CQ blood levels both in intervention and control children during follow-up. However, the effects of another community based malaria study which was implemented in all villages of the rural CRSN study and during which all newborn children received insecticide-treated mosquito nets (ITN) free of charge may also play a role in this overall dynamic [21]. As the enrolment of such study children took place from mid-2000 until the end of 2002, increasing protection of under five children with ITN is a likely additional explanation for the observed reductions of malaria morbidity in both the intervention and control villages of the MAMOP study. However, the design and conduct of the ITN trial makes it very unlikely that there was a differential effect on the MAMOP study groups.
Moreover, the observed differences in malaria parameters between the two surveys may also be explained by differences in malaria transmission intensities due to annual variations in the pattern and amount of rainfall.

Another important observation from this study is the significant increase in clinically relevant $C Q$ resistance in the study area over the two years observation period. This finding supports similar findings from a number of other studies which looked at the efficacy of CQ in Burkina Faso in recent years $[18,19]$. However, the fact that a more intense CQ drug pressure in the intervention villages was not associated with a more rapid increase of in vivo CQ resistance lends support to the hypothesis that better compliance with the full course of antimalarial treatment will likely delay resistance development $[12,29]$.

Obviously, all-cause mortality and cause-specific mortality would have been the best outcome measure for this kind of intervention study. Due to sample size considerations and costs moderate to severe anaemia was chosen as the primary endpoint. The MAMOP community intervention had no effect on the prevalence of anaemia. This could at least partly be explained by the finding from another cohort study in the rural CRSN study area which has demonstrated that malnutrition but not malaria is the main determinant of anaemia development in young children of the area [30]. However, as the MAMOP study showed also no effects on other malaria parameters the likely explanation is that the intervention was simply not effective due to contamination, pre-existing differences in the coverage of malaria treatment in both groups and the unexpected rapid development of CQ resistance in the area.

Only a few studies have tried to measure the effects of complex malaria treatment interventions at the community level in endemic areas. One study in Ethiopia has measured the effectiveness of treating malaria episodes of young children through their mothers on mortality and was able to show a major reduction in all-cause mortality and malaria-specific mortality attributed to the interven- 
tion [15]. In a comparable study in Burkina Faso the intervention was associated with a significant reduction in malaria morbidity, but mortality was not measured [14]. However, other studies conducted in Kenya, The Gambia and Zaire and which were using village health volunteers for community malaria treatment were unable to show sufficient effects of the intervention on morbidity and mortality in young children [31-34].

In conclusion, this study has shown the feasibility of a complex malaria community intervention which has bridged the gap between the health workers at the peripheral health centres and the mothers of young children in individual households through women groups. The coverage of malaria treatment has substantially improved during the trial. Although such an approach is promising given the continuous lack of access to formal health services in much of rural SSA, there was no major difference in effectiveness between intervention and control areas. A possible intervention effect may have been masked by contamination, pre-existing differences in the coverage of malaria treatment in both groups, and the rapid rise in CQ resistance in the study area. Future studies should try to avoid confounding influences of other malaria interventions, employ more effective malaria first-line drugs such as artemisinin-based combination therapies (ACT), and preferably use mortality as the primary endpoint.

\section{Authors' contributions}

$\mathrm{BK}, \mathrm{FS}, \mathrm{AJ}, \mathrm{OM}, \mathrm{RS}$ and GT designed the study. FS, BC and $\mathrm{BK}$ were responsible for the conduct of the study in Burkina Faso. FS, OM and HB analysed the data. All authors contributed to the interpretation of the data, helped write the paper, and read and approved the final manuscript.

\section{Acknowledgements}

The study was funded by EU INCO-DEV (project no IC A4-CT-200I10010). The coordinator of this study, Florent Somé, tragically died in August 2005. This paper would not have been possible without his dedication and his untiring work during the development and implementation of the study.

\section{References}

I. Greenwood B, Bradley A, Greenwood A, Byass P, Jammeh K, Marsh $\mathrm{K}$, Tulloch S, Oldfield F, Hayes R: Mortality and morbidity from malaria among children in a rural area of The Gambia. Trans $R$ Soc Trop Med Hyg 1987, 81 : 478-486.

2. WHO: World malaria situation in 1994. Wkly Epidemiol Rec 1997, 72:269-92.

3. Snow RW, Craig M, Deichmann U, Marsh K: Estimating mortality, morbidity and disability due to malaria among Africa's nonpregnant population. Bull World Health Organ 1999, 77:624-640.

4. Müller O, Traoré C, Kouyaté B, Becher H: Malaria morbidity, treatment seeking behaviour, and mortality in a cohort of young children in rural Burkina Faso. TM \& IH 2003, 8:290-296.

5. Nsimba SED, Warsame M, Tomson G, Massele AY, Mbatiya ZA: A household survey of source, availability, and use of antimalarials in a rural area of Tanzania. Drug Information Journal 1999 , 33:1025-32.
6. McCombie SC: Self-treatment for malaria: the evidence and methodological issues. Health Policy Plan 2002, 17:333-344.

7. Krause G, Sauerborn R: Community-effectiveness of care - the example of malaria treatment in rural Burkina Faso. Ann Trop Paediatr 2000, 7:99-106.

8. Okonkwo PO, Akpala CO, Okafor HU, Mbah AU, Nwaiwu O: Compliance to correct dose of chloroquine in uncomplicated malaria correlates with improvement in the condition of rural Nigerian children. Trans $R$ Soc Trop Med Hyg 200I, 95:320-24.

9. Trapé $\mathrm{J}$ : The public health impact of chloroquine resistance in Africa. Am J Trop Med Hyg 200I, 64(suppl): I2-17.

10. Müller $O$, Razum $O$, Traore $C$, Kouyate $B$ : Community effectiveness of chloroquine and traditional remedies in the treatment of young children with falciparum malaria in rural Burkina Faso. Malar J 2004, 3:36.

II. Nsungwa-Sabiiti J, Tomson G, Pariyo G, Ogwal-Okeng J, Peterson S: Community effectiveness of malaria treatment in Uganda a long way to Abuja targets. Ann Trop Paediatr 2005, 25:89-98.

12. Greenwood BM, Bojang K, Whitty JM, Targett GA: Malaria. The Lancet 2005, 365: | 487-98.

13. Newton PN, Green MD, Fernández FM, Day NPJ, White NJ: Counterfeit anti-infective drugs. Lancet Infect Dis 2006, 6:602-6I3.

14. Pagnoni F, Convelbo N, Tiendrebeogo J, Cousens S, Esposito F: A community-based programme to provide promt and adequate treatment of presumptive malaria in children. Trans $R$ Soc Trop Med Hyg 1997, 91:5 12-17.

15. Kidane G, Morrow RH: Teaching mothers to provide home treatment of malaria in Tigray, Ethiopia: a randomised trial. The Lancet 2000, 356:550-55.

16. Müller O, Becher H, Baltussen A, Ye Y, Diallo D, Konate M, Gbangou A, Kouyate B, Garenne M: Effect of zinc supplementation on malaria morbidity among West African children: a randomized double-blind placebo-controlled trial. BM] 200I, 322: $1567-1572$

17. Traoré C: Epidemiology of malaria in a holoendemic area of rural Burkina Faso. In PhD thesis University of Heidelberg; 2003.

18. Kouyaté B, Sie A, Yé M, De Allegri M, Müller O: The great failure of malaria control in Africa: a view from a district in Burkina Faso. PLoS med 2007, 4:el 27.

19. Müller O, Traoré C, Kouyaté B: Clinical efficacy of chloroquine in young children with uncomplicated malaria - a community based study in rural Burkina Faso. TM \& IH 2003, 8:202-203.

20. Mene Miaffo C, Somé F, Kouyaté B, Jahn A, Müller O: Malaria and anemia prevention in pregnant women of rural Burkina Faso. BMC Pregnancy and Childbirth 2004, 4: 18.

21. Müller O, Traoré C, Kouyaté B, Yé Y, Frey C, Coulibaly B, Becher H Effects of insecticide-treated bed net protection during early infancy in an African area of intense malaria transmission: randomized controlled trial. Bull World Health Organ 2006, 84: $120-26$.

22. Okrah J, Traoré C, Palé A, Sommerfeld J, Müller O: Community factors associated with malaria prevention by mosquito nets: an exploratory study in rural Burkina Faso. TM \& IH 2002, 7:240-248.

23. Sommerfeld J, Sanon M, Kouyaté B, Sauerborn R: Informal risksharing arrangements in rural Burkina Faso: implications for community-based health care and insurance. Hum Organ 2002, 61:139-46.

24. Kynast-Wolf G, Sankoh OA, Gbangou A, Kouyate B, Becher H: Mortality patterns, 1993-98, in a rural area of Burkina Faso, West Africa, based on the Nouna demographic surveillance system. TM \& IH 2002, 7:349-56.

25. WHO: Assessment and monitoring of antimalarial drug efficacy for the treatment of uncomplicated falciparum malaria. WHO/HTM/RBM/2003.50

26. Campbell MK, Thomson S, Ramsey CR, McLennan GS, Grinshaw JM: Sample size calculator for cluster randomized trials. Comput Biol Med 2004, 34: I I 3- 125.

27. Ansah EK, et al:: Improving adherence to malaria treatment for children: the use of pre-packed chloroquine tablets vs. chloroquine syrup. TM \& IH 200I, 6:496-504.

28. Yeboah-Antwi K, et al:: Impact of prepacking antimalarial drugs on cost to patients and compliance with treatment. Bull World Health Organ 200I, 79:394-399. 
29. Talisuna AO, Bloland P, D'Alessandro U: History, dynamics, and public health importance of malaria parasite resistance. Clin Microbiol Rev 2004, I7:235-254.

30. Müller O, Traoré C, Jahn A, Becher $\mathrm{H}$ : Severe anaemia in west African children: malaria or malnutrition? The Lancet 2003, 36I:86-87.

31. Spencer HC, Kaseje DCO, Mosley WH, Sempebwa EKN, Huong AY, Roberts JM: Ann Trop Med Parasitol 1987, 8 I (suppl):36-45.

32. Greenwood BM, Greenwood AM, Bradley AK, Snow RW, Byass P, Hayes RJ, N'Jie $A B H$ : Comparison of two strategies for control of malaria within a primary health care programme in The Gambia. The Lancet 1988, 330: I I2 I-27.

33. Greenwood BM, Bradley AK, Byass P, Greenwood AM, Menon A Snow RW, Hayes RJ, Hatib-N'Jie AB: Evaluation of a primary health care programme in The Gambia. II Its impact on mortality and morbidity in young children. J Trop Med Hyg 1990, 93:87-97.

34. Delacollette C, Van der Stuyft P, Molima K: Using community health workers for malaria control: experience in Zaire. Bull World Health Organ 1996, 74:423-30.

Publish with Biomed Central and every scientist can read your work free of charge

"BioMed Central will be the most significant development for disseminating the results of biomedical research in our lifetime. "

Sir Paul Nurse, Cancer Research UK

Your research papers will be:

- available free of charge to the entire biomedical community

- peer reviewed and published immediately upon acceptance

- cited in PubMed and archived on PubMed Central

- yours - you keep the copyright

Submit your manuscript here:

http://www.biomedcentral.com/info/publishing_adv.asp
BioMedcentral 